\title{
FINITE ELEMENT MODELLING FOR TENSILE BEHAVIOUR OF THERMALLY BONDED NONWOVEN FABRIC
}

\author{
Xiaoping Gao*, Liping Wang \\ Inner Mongolia University of Technology, College of Light Industry and Textile, Hohhot, Inner Mongolia 010080, China \\ +864713603443 gaoxp@imut.edu.cn
}

\begin{abstract}
:
A nonwoven fabric has been widely used in geotextile engineering in recent years; its tensile strength is an important behaviour. Since the fibre distributions in nonwoven fabrics are random and discontinuous, the unit-cell model of a nonwoven fabric cannot be developed to simulate its tensile behaviour. This article presents our research on using finite element method (FEM) to study the tensile behaviour of a nonwoven fabric in macro-scale based on the classical laminate composite theory. The laminate orientation was considered with orientation distribution function of fibres, which has been obtained by analysing the data acquired from scanning electron microscopy with Hough Transform. The FE model of a nonwoven fabric was developed using ABAQUS software; the required engineering constants of a nonwoven fabric were obtained from experimental data. Finally, the nonwoven specimens were stretched along with machine direction and cross direction. The experimental stress-strain curves were compared with the results of FE simulations. The approximate agreement proves the validity of an FE model, which could be used to precisely simulate the stress relaxation, strain creep, bending and shear property of a nonwoven fabric.
\end{abstract}

\section{Keywords:}

Tensile Behaviour; Finite Element Analysis; Nonwoven Fabric; Classic Laminate Composite Theory

\section{Introduction}

Since the nonwoven geotextiles are of considerable interest in their satisfactory performance as constructional materials, it has been employed in many applications, such as embankments on weak foundations, railroad track structures, etc. In contrast to knitted fabric and woven fabric, the random and discontinuous microstructure is the most characteristic features of a nonwoven fabric, which has an important effect on its tensile behaviour [1].

Tensile behaviour is the most common method in characterizing the mechanical behaviour of the nonwoven fabric. Due to the complex structure of nonwoven fabrics, a few researchers had used considerable efforts to understand the mechanical behaviour of the nonwoven fabric by applying the mechanical model. For example, Rawal et al. [1] developed a micromechanical model by incorporating the effect of fibre orientation. Hou et al. [2] investigated the effects of random and discontinuous microstructure of the nonwoven fabric on their mechanical properties by incorporating random discontinuous structures representing micro-structures of a real nonwoven material. Ridruejo et al. [3] studied the mechanical behaviour and related these behaviours with the property of individual fibre. Gautier et al. [4] studied the anisotropic mechanical behaviour of nonwoven geotextiles with tensile test. Kim [5] established a relationship between geometrical features and mechanical performance of point-bonded nonwoven fabrics.

FEAapproach was usually used to simulate the tensile behaviour of thermally bonded nonwoven fabrics; a few studies [6-11] were performed to construct a numerical model and predict the mechanical response of the nonwoven fabric. In FEA, the solidified area within the bonding point was represented by solid elements, and the connecting fibres [9] between the bonded areas were modelled with linking element. Limem and Warner [10] treating the nonwoven material as a 2D structure composed truss elements and isotropic planar elements representing fibre matrix and bond point, respectively. The fibres and bond points were modelled using shell elements with orthotropic material properties assigned to the structure [11]. The behaviour of a single fibre and the anisotropic structure of the bond points and matrix were incorporated into the model, which was confirmed by tensile results. However, it has the limitation that the structure had a large number of fibres. Smita et al. [12] modelled the non-uniform deformation of the spunbonded nonwoven fabric by applying the finite element model and calculated the variation of Poisson's ratio with longitudinal strains.

The finite element method (FEM) has been realized to be one of the most powerful tools in analysing stress change and computing the independent variation of stress on time. However, their applicability to random structures of the nonwoven fabric is restricted. As is well known, it is difficult to develop a unitcell model for describing nonwoven performance due to a random structure of nonwoven materials and the nonlinear material property of single fibre. In this manner, the smallest entity of the structure and the single fibre have to be divided into a large number of elements. The large number of elements would lead to a very large system of equations resulting in very long solution times of the system. Thus, the idea that direct 
application of FEM to the task of modelling nonwoven would lead to difficulties.

In this paper, a FE model for characterizing the tensile behaviour of a thermally bonded nonwoven fabric was developed by using ABAQUS ${ }^{\circledR}$ software based on the classical laminate composite theory by treating the fibrous networks as orthotropic and layered structure materials. To allow comparability of tensile behaviours of the nonwoven fabric in two principal directions with the tensile tests, the boundary conditions implemented in the FE model were identical to those used in the tensile tests. Finally, nonwoven specimens were subjected to tensile loading along with machine direction and cross direction. The theoretical results were compared with the experimental ones.

\section{Material Properties}

The thermally bonded nonwoven fabric is manufactured by using polypropylene fibres (1.56dtex, $38 \mathrm{~mm}$ ) by applying the heat energy to the thermoplastic component present in fibrous web and the polymer flows by surface tension and capillary action to form fibrous network. The nominal thickness of a nonwoven fabric is $0.42 \mathrm{~mm}$ and its density is $150 \mathrm{~g} / \mathrm{m}^{2}$. Since the thickness is very small compared with lateral dimensions of the specimen, the nonwoven was assumed as $2 \mathrm{D}$ structures. The scanning electron microscopic (SEM) image of the nonwoven fabric structure is shown in Figure 1.

The material constants [13] required in finite element modelling such as Young's modulus and Passion's ratios were defined based on the classic composite theory, and the orthotropic mechanical parameters were calculated from the standard tensile experiment.

Due to the non-uniform microstructure and material properties of the fabric, different sizes of specimens may indicate different types of material behaviour. The mechanical properties of the nonwoven fabrics were tested using INSTON Universal Testing Machine under standard laboratory condition $\left(20^{\circ} \mathrm{C}, 65 \%\right.$ relative humidity).
Nonwoven tensile tests were carried out using 10 nonwoven specimens (300 mm $\times 50 \mathrm{~mm}$ dimension) at a gauge of 200 $\mathrm{mm}$ clamp distance and a speed of $100 \mathrm{~mm} / \mathrm{min}$, and thus the deformation rate is $0.008 \mathrm{1} / \mathrm{s}$. The tensile directions were $0^{\circ}$ and $90^{\circ}$ with respect to machine direction of the fabric. The maximum stress and the elongation at maximum stress were obtained from the load elongation data. The secant modulus, i.e. Young's modulus of the fabric, was obtained by applying the first derivative of the stress-strain curve at $10 \%$ elongation. The typical stress-strain curves for nonwoven material in machine direction and cross direction are shown in Figure 2.

The secant modulus in CD and MD was computed from stressstrain curves (shown in Figure 2) with Equation (1), in which the thickness of the material was assumed as constant

$$
E=\Delta \sigma / \Delta \varepsilon
$$

where $\Delta \sigma$ is the incremental stress (MPa) and $\Delta \varepsilon$ is the net strain during stretch (\%).

With respect to the method [14], we can deduce the function for calculating the shear modulus at different shear directions by applying the orthotropic theory to nonwoven fabric.

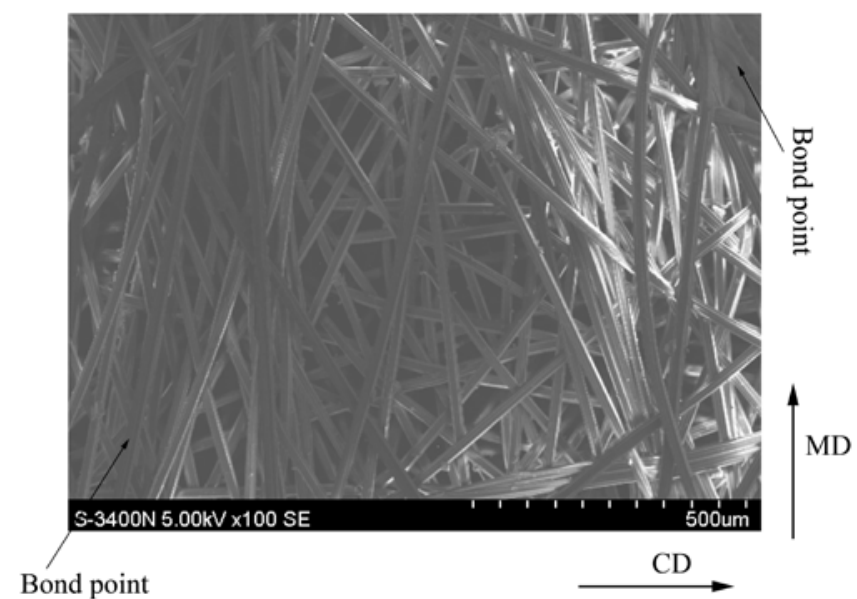

Figure 1. SEM image of a nonwoven fabric.
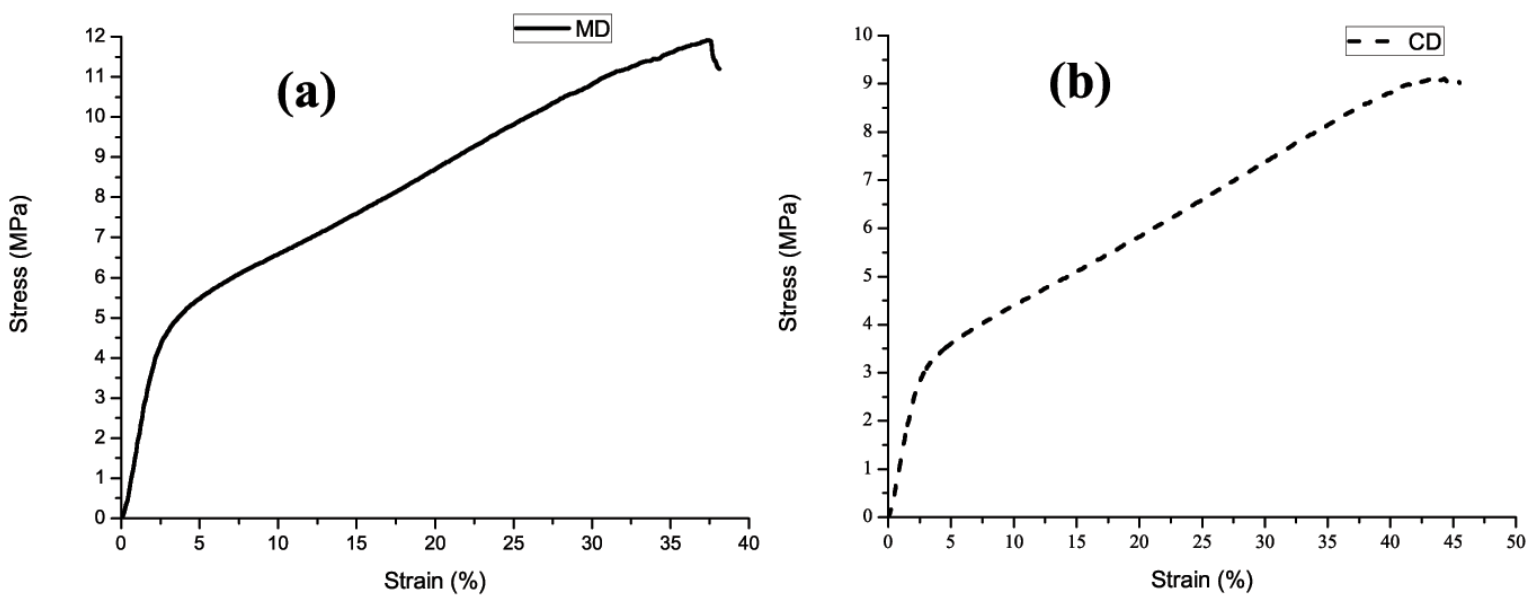

Figure 2. The typical stress-strain curves of a nonwoven fabric: (a) MD (machine direction) and (b) CD (cross direction). 


$$
\frac{1}{E_{\theta}}=\frac{1}{E_{1}}(\cos \theta)^{4}+\left(\frac{1}{G_{12}}-\frac{2 v_{12}}{E_{1}}\right)(\sin \theta \cos \theta)^{2}+\frac{1}{E_{2}}(\sin \theta)^{4}
$$

where $E_{\theta}$ is the tensile modulus along $\theta$ direction, $E_{1}$ is the tensile modulus in MD, $E_{2}$ is the tensile modulus in CD, $G_{12}$ is the shear modulus in-plane, and $v_{12}$ is the Poisson ratio inplane.

When $\theta$ equals to $45^{\circ}$, the shear modulus could be deduced as follows:

$$
\frac{1}{G_{12}}=\frac{4}{E_{45}}+\frac{2 v_{12}}{E_{1}}-\frac{1}{E_{1}}-\frac{1}{E_{2}}
$$

Thus, the stiffness constants of the nonwoven fabric could be calculated from Equations (1)-(3).

Poisson's ratio of the nonwoven fabric could be calculated from the deformation ratio of longitudinal vs. transverse, which was assumed as 0.41 in two directions [2] in this paper.

The transverse shear modulus and Poisson's ratio were characterized by the following equations:

$$
\begin{aligned}
G_{i j} & =\frac{E_{i}}{2\left(1+v_{i j}\right)} \\
\frac{E_{i}}{v_{i j}} & =\frac{E_{j}}{v_{j i}}
\end{aligned}
$$

In summary, the parameter values $E_{1}, E_{2}, G_{12}$ and $v_{12}$, which were used to characterize the performance of fabric, were measured experimentally. The computed modulus and Poisson's ratio are shown in Table 1.

\section{Estimation of the fibre orientation and frequency distribution}

The fibre orientation distribution was estimated using either fast Fourier transform or Hough transform (HT) methods. The advantage of the HT method [15] is that the actual orientation of the lines can be calculated directly from the result of the transform without the need for any further computation. In FEA, the non-uniformity was quantified with respect to the orientation distribution function (ODF), which could be obtained by applying $\mathrm{HT}$ to the SEM and used for calculation of the orthotropic parameters.

Therefore, a new algorithm based on the HT was written in Matlab $^{\circledR}$ to compute the ODF of the fibre for SEM images of the fibres. The ODF of the thermally bonded nonwoven fabric was calculated based on the SEM image and presented in Figure 3.

\section{Finite Element Analysis}

\subsection{FE Model}

In this article, although the structure of the nonwoven fabric has been a discontinuity in contrast to most standard structural materials, a macro-scale continuum FE model for nonwoven fabric was developed in $\mathrm{ABAQUS}^{\circledR}$ based on the classical composite theory by treating the fibrous network as the orthotropic material and used to simulate the uniaxial stretched stress-strain. The density of the nonwoven fabric is $1.2 \times 10^{-9}$ ton $\mathrm{mm}^{-3}$.

Experimentally measured elastic material properties of the nonwoven fabric were introduced into the models. The nonwoven web was assumed to be made up of three layers of fibres such that all fibres in a layer were oriented in the same direction according to the ODF of fibre, as shown in Figure 4.

The FE model of a nonwoven specimen was developed on the basis of the commercial finite element software with dimensions of $30 \mathrm{~mm} \times 15 \mathrm{~mm}$, and the commercial FE software ABAQUS/ Standard was used to simulate the tensile deformation of nonwoven.

\subsection{Boundary Condition}

The nonwoven fabric was modelled as a rectangular plate and fixed at its one edge (the boundary condition of the fixed edge was defined as 'ENCASTRE' in ABAQUS ${ }^{\circledR}$ ). The boundary conditions were set to emulate the holding of the nonwoven fabric. To allow comparability of tensile behaviours of the nonwoven fabric in two principal directions with the tensile tests, the boundary conditions, i.e. the constant loading speed implemented in the

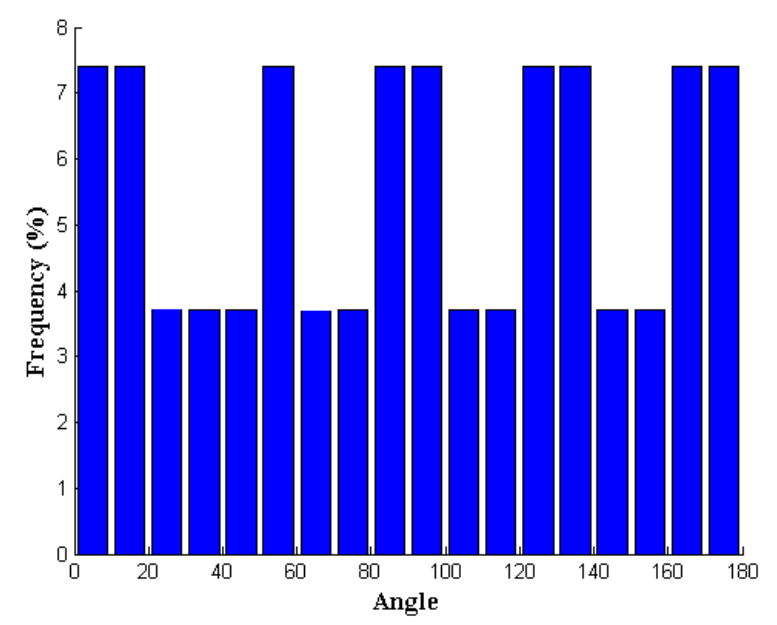

Figure 3. The ODF of the thermally bonded nonwoven fabric.

Table 1. Mechanical properties of a nonwoven fabric.

\begin{tabular}{|c|c|c|c|c|c|c|}
\hline Nonwoven & E11 (MPa) & E22 (MPa) & G12 (MPa) & G13 (MPa) & G23 (MPa) & $v_{12}$ \\
\hline Thermal bonding & 357.59 & 140.04 & 126.80 & 126.80 & 49.66 & 0.41 \\
\hline
\end{tabular}




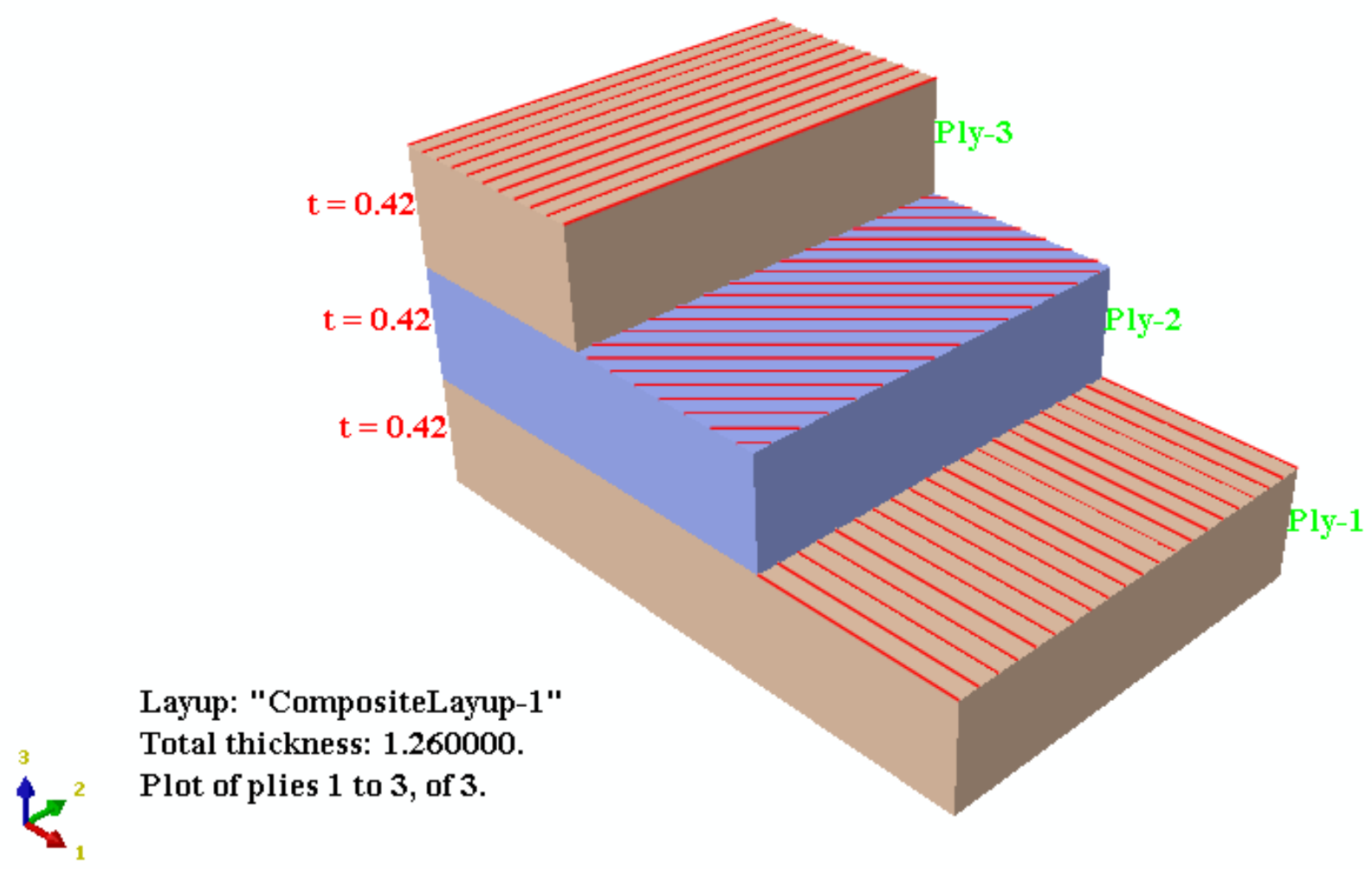

Figure 4. Schematic diagram of three layers in an FE model.

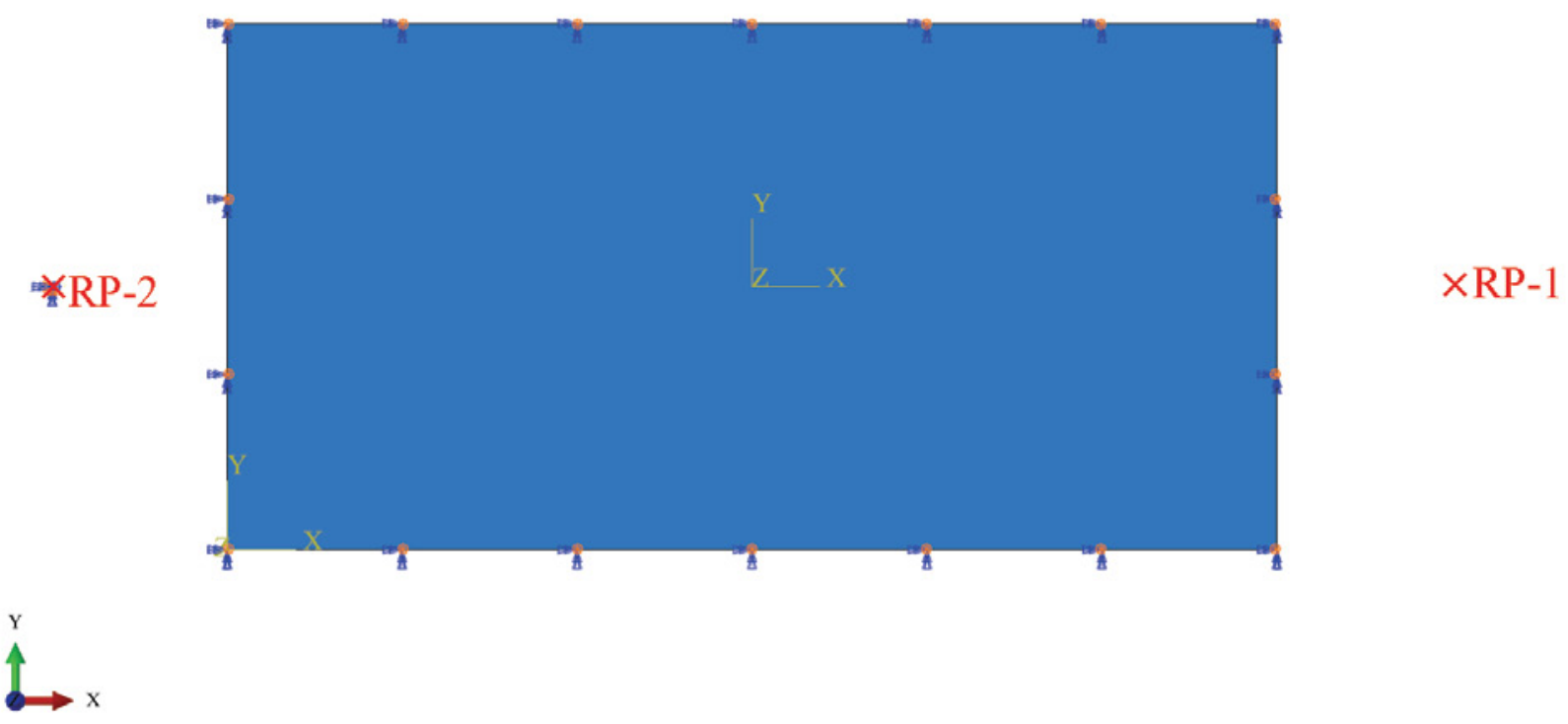

Figure 5. A set of boundary conditions.

FE model was identical to those used in tensile tests, were applied to the opposite boundary of the models to simulate the static tensile deformation, as shown in Figure 5.

\subsection{Meshing}

Due to stability issues and finite strain plasticity ineligibility, membrane elements were not preferred for the model. The model of the nonwoven specimen with dimensions of $30 \mathrm{~mm}$ $\times 15 \mathrm{~mm}$ consists of 1800 three-node triangular thin shell elements (S3R shell element, triple-node triangular element taking into account the fibrous structure of the nonwoven through the fibre orientation distribution density. This element allows us to describe the tensile behaviour of the shell under large deformation).

The thickness is very small compared with the lateral dimensions of the specimen. The assumption of plane stress 
is a simplification of three-dimensional elasticity problems, and thus stresses in the z-direction are negligibly small.

\subsection{Simulation Results and Experiment Verification}

The fracture deformation of the nonwoven fabric in MD was shown in Figure 6. The FE result and experimental data for both MD and CD models were shown in Figure 7.

The predicted stress-strain during tensile with FEM were compared with that obtained from experiments in MD and CD. As obvious from Figure 7, there is an approximate agreement between FE simulation results and the experimental result. The maximum ratio of errors sum of squares to experimental result in MD and CD direction are $24.9 \%$ and $19.7 \%$, respectively. The difference between experimental results and numerical results was attributed to fact that the fibre actual distribution was not accurately modelled in the model. At the same time, the slight deviation of the numerical results from experimental results could be explained as its continuous nature of the model could not include the discontinuous and non-uniform microstructure of the material.

The difference in the initial stages of deformation is due to the underestimation of the real stress by the continuous FEA model since it could not account for deformation mechanisms acting in nonwovens at micro-scale. The maximum stress in numerical simulation is slightly higher than the experimental data under the same strain. Despite some differences existing between FE results and experimental data, the FE model developed in this paper could effectively be used to simulate the tensile property of the nonwoven fabric.
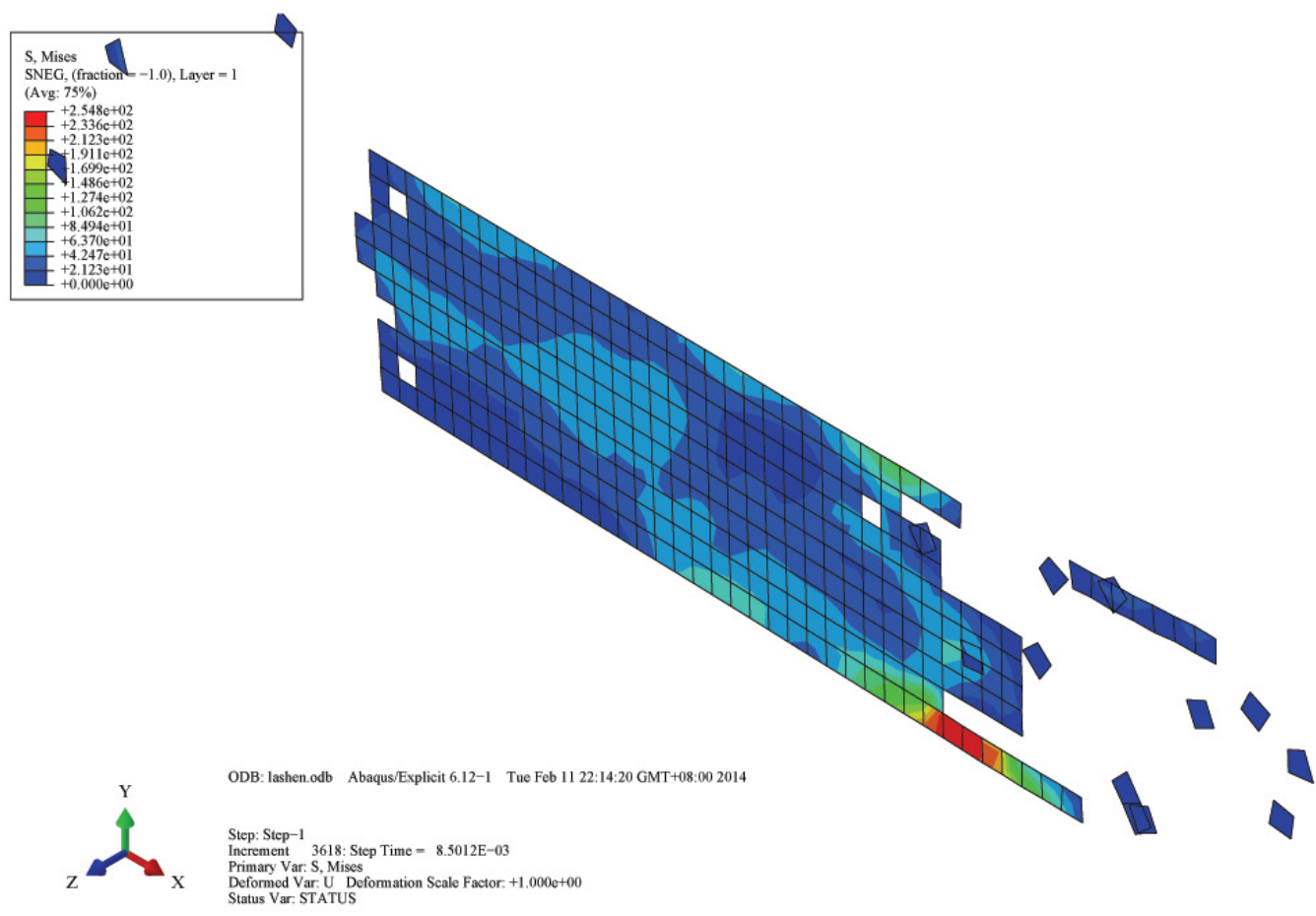

Figure 6. Finite element model for deformation of the nonwoven fabric after $19.6 \%$ extension in MD.
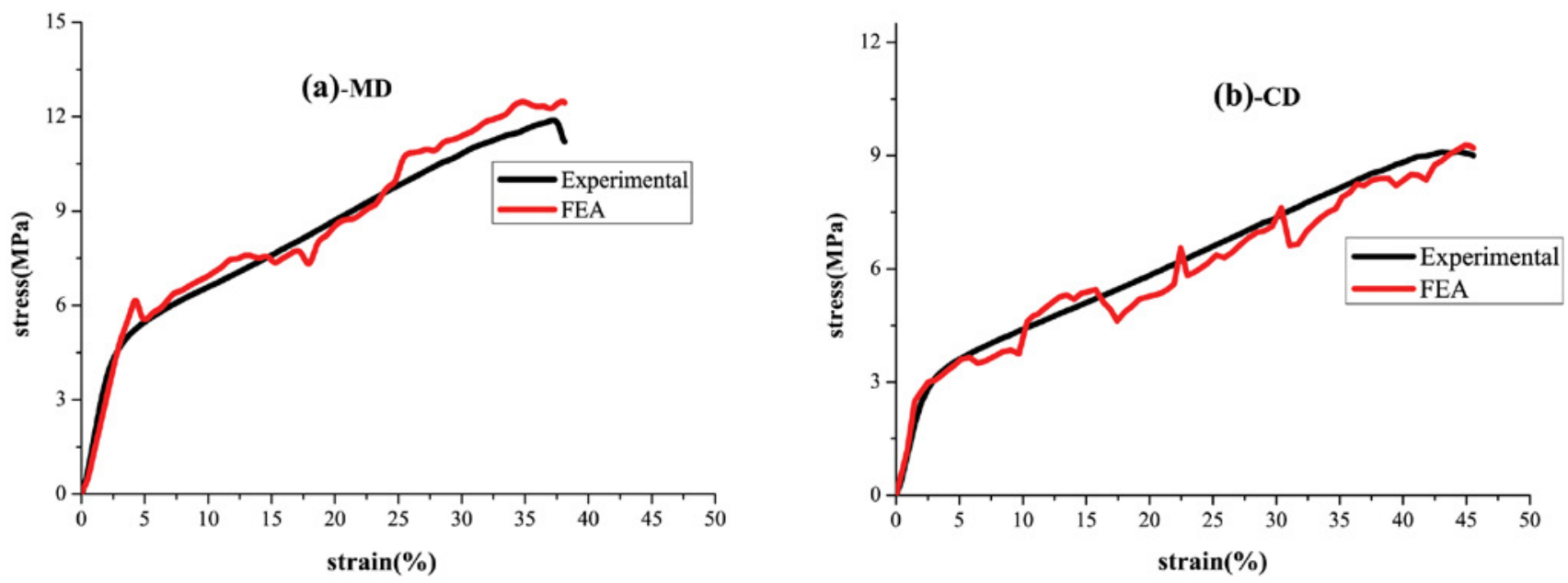

Figure 7. The FE result vs. experimental results. 


\section{Conclusions}

In this paper, the tensile behaviour of the nonwoven fabric in macro-scale was studied by using FEM. The laminate orientation was considered with ODF of fibres, which was obtained by analysing the data acquired from SEM with HT. An FE model has been developed in ABAQUS $\otimes$ software based on the classical lamination theory to characterize the tensile behaviour of the nonwoven fabric. The nonwoven specimen was subjected to tensile tests along machine direction and cross direction. The experimental stress-strain curve was compared with the results of FE simulations. It was found that there is an approximate agreement, which proves the validity of the FE model. Thus, the FE model could be used to precisely simulate the stress relaxation, strain creep property, bending and shear property of the nonwoven fabric.

\section{Acknowledgement}

The authors acknowledge the financial supports from the Natural Science Foundation of Inner Mongolia Autonomous Region of China (Grant No. 2012MS0811) and the Foundation of Inner Mongolia University of Technology (Grant No.ZD201419).

\section{References}

[1] Rawal A, Priyadarshi A, Kumar N, Lomov S.V, Verpoest I. Tensile behaviour of nonwoven structures: comparison with experimental results. (2010). Journal of Materials Science, 45(24): 6643-6652.

[2] Hou X.N, Acar M, Silberschmidt V.V. Finite element simulation of low-density thermally bonded nonwoven materials: Effects of orientation distribution function and arrangement of bond points.(2011). Computational Materials Science, 50(4): 1292-1298.

[3] Ridruejo A, González C, Llorca J. Micromechanisms of deformation and fracture of polypropylene nonwoven fabrics.(2011). International Journal of Solids and Structures, 48(1): 153-162.
[4] Gautier K.B, Kocher C.W, Drean J.Y. Anisotropic mechanical behavior of nonwoven geotextiles stressed by uniaxial tension. (2007). Textile Research Journal, 77(1): 20-28.

[5] Kim H.S. Relationship between fiber orientation distribution function and mechanical anisotropy of thermally pointbonded nonwovens. (2004). Fibers and Polymers, 5(3): 177-181.

[6] Demirci E, Acar M, Pourdeyhimi B, Silberschmidt V.V. Computation of mechanical anisotropy in thermally bonded bicomponent fibre nonwovens. (2012). Computational Materials Science, 52(1): 157-163.

[7] Hou X.N, Acar M, Silberschmidt V.V. 2D finite element analysis of thermally bonded nonwoven materials: Continuous and discontinuous models.(2009). Computational Materials Science, 46(3): 700-707.

[8] Villard P, Chevalier B, Hello L.B, Combe G. Coupling between finite and discrete element methods for the modelling of earth structures reinforced by geosynthetic. (2009). Computers and Geotechnics, 36(5): 709-717.

[9]Mueller D.H, Kochmann M.M. Neumerical Modeling of Thermobonded Nonwovens. (2004) International Nonwovens Journal, 13(1): 56-62.

[10] Limem S, Warner S.B. Adhesive point-bonded spunbond fabrics.(2005). Textile Research Journal, 75(1): 63-72.

[11] Demirci E, Acar M, Pourdeyhimi B, Silberschmidt V.V. Finite element modelling of thermally bonded bicomponent fibre nonwovens: Tensile behaviour.(2011) Computational Materials Science, 50(4): 1286-1291.

[12] Smita B.S, Sherrill B.B, Goswami B.C. Finite element modeling of the nonuniform deformation of spun-bonded nonwovens. (1998). Textile Research Journal, 68(5): $327-$ 342.

[13] Backer S, Petterson D.R. Some Principles of Nonwoven Fabrics.(1960). Textile Research Journal, 30(9): 704-711.

[14] Kim H.S. Orthotropic theory for the prediction of mechanical performance in thermally point-bonded nonwovens. (2004). Fibers and Polymers, 5(2): 139-144.

[15] Xu B.G, Yu L. Determining fiber orientation distribution in nonwovens with Hough transform techniques.(1997). Textile Research Journal, 67(8): 563-571. 